\title{
Cross-species gene normalization by species inference
}

\author{
Chih-Hsuan $\mathrm{Wei}^{\dagger},{ }$ Hung-Yu Kao ${ }^{* \dagger}$ \\ From The Third BioCreative - Critical Assessment of Information Extraction in Biology Challenge \\ Bethesda, MD, USA. 13-15 September 2010
}

\begin{abstract}
Background: To access and utilize the rich information contained in the biomedical literature, the ability to recognize and normalize gene mentions referenced in the literature is crucial. In this paper, we focus on improvements to the accuracy of gene normalization in cases where species information is not provided. Gene names are often ambiguous, in that they can refer to the genes of many species. Therefore, gene normalization is a difficult challenge.
\end{abstract}

Methods: We define "gene normalization" as a series of tasks involving several issues, including gene name recognition, species assignation and species-specific gene normalization. We propose an integrated method, GenNorm, consisting of three modules to handle the issues of this task. Every issue can affect overall performance, though the most important is species assignation. Clearly, correct identification of the species can decrease the ambiguity of orthologous genes.

Results: In experiments, the proposed model attained the top-1 threshold average precision (TAP-k) scores of $0.3297(k=5), 0.3538(k=10)$, and $0.3535(k=20)$ when tested against 50 articles that had been selected for their difficulty and the most divergent results from pooled team submissions. In the silver-standard-507 evaluation, our TAP-k scores are 0.4591 for $k=5,10$, and 20 and were ranked $2^{\text {nd }}, 2^{\text {nd }}$, and $3^{\text {rd }}$ respectively.

Availability: A web service and input, output formats of GenNorm are available at http://ikmbio.csie.ncku.edu.tw/ GN/.

\section{Background}

In recent years, the amount of biological literature has increased rapidly. Text-mining techniques for extracting information from this work are not completely reliable [1]. Extracting information on proteins automatically and precisely is very important and difficult. Many methods have been developed, and they mainly consist of two tasks. Relation extraction identifies the relationships among biomedical entities in the literature. In extracting relations, each biomedical entity, such as a gene, protein or disease, in an article is mapped to its database identifier. This task is called name entity

\footnotetext{
* Correspondence: hykao@mail.ncku.edu.tw

+ Contributed equally

Department of Computer Science and Information Engineering, National

Cheng Kung University, Tainan, Taiwan, R.O.C

Full list of author information is available at the end of the article
}

normalization. This is particularly challenging given the high ambiguity in biology and biomedicine of many entity names, such as the gene/protein name [2-7], species name [8-10], and the chemical/compound name $[11,12]$.

The Critical Assessment of Information Extraction Systems in Biology (BioCreative), a renowned competition in the field of biological text mining, covers a variety of important issues. BioCreative III addressed three text-mining tasks in the domain of molecular biology: gene normalization $(\mathrm{GN})$, protein-protein interactions (PPI), and an interactive demonstration task for gene indexing and retrieval (IAT). The GN task in BioCreative III was similar to the same tasks in previous BioCreative competitions [4-6], in that the goal was to map genes or proteins mentioned in the literature to standard database identifiers. The GN tasks of BioCreative 
II.5 \& III were more difficult than those in preceding challenges. In particular, species information was not provided, and the normalization targets were changed to full-text articles. The GN tasks of BioCreative I \& II had been limited to a specific species, such as a human, fly, yeast, mice, and the tasks extracted information from abstracts in the literature. These two differences resulted in a more challenging evaluation than the previous competitions.

Many gene normalization studies have focused on GN tasks in which species information is provided. Hakenberg [13] developed an outstanding gene-name normalization system, winning the BioCreative II name entity normalization task. ProMiner [3] is a well-known genename normalization system that employs a dictionarybased approach and relies on manual curation. GENO [7] is a high-performing and efficient gene-name normalization system. It applies the TF-IDF weighting scheme and calculates semantic similarity scores to resolve ambiguous terms. All of these systems perform well, obtaining F-measures of $80 \%$. Later, Hakenberg [2] developed a cross-species normalization system, GNAT, which considers 13 different species and obtains an Fmeasure of $81.4 \%$.

The interactor normalization task (INT) of BioCreative II.5 did not provide species information and focused on full-text articles. Owing to the two difficult characteristics of this task, the normalization results might seem surprisingly low [5]. Hakenberg et al [14] modified their previous work, including GNAT and a gene mention recognition system (BANNER), and obtained the highest precision (F-measure 43.4\%). They disambiguated species and assigned candidate identifiers to proteins mentions. Chen et al. [15] developed a Biological Literature Miner (BioLMiner) system to handle the INT and IPT(interaction pair task) tasks. Two of their subsystems involved in the INT task are the gene mention recognizer (GMRer) and a gene normalizer (GNer). These two subsystems were developed based on support vector machine (SVM) and a conditional random field with designed informative features. Verspoor et al. [16] introduced an approach using fuzzy dictionary lookup to detect mentions of proteins. They also described several strategies for disambiguating species associated with gene mentions; these strategies operated globally (throughout the document) and locally (in the immediate vicinity of a protein mention). Sætre et al. [17] used many subcomponents to produce the AkaneRE system, which obtained the highest recall (68.3\%). The AkaneRE is provided by the U-Compare system, and it includes sentence boundary detection, tokenization, stemming, part-of-speech tagging, parsing, named-entity recognition, generation of potential relations, generation of features for each relation, and finally, assignment of confidence scores and ranking of candidate relations. Dai et al. [18] used a three-stage normalization algorithm with a ranking method to handle this task. For interactor ranking, candidate identifiers are ranked by SVM classifier with baseline features and template features.

The purpose of the GN task in BioCreative III is to produce a list of the EntrezGene [19] identifiers of all species for gene mentions in full-text articles. This task is a complicated challenge involving three issues: genemention variation, orthologous gene ambiguity and intra-species gene ambiguity. Gene-mention variation occurs when a gene in a dictionary has multiple names. Gene names in the literature also show high variation, including orthographical variation (e.g., "TLR7" and "TLR-7"), morphological variation (e.g., "GHF-1 transcriptional factor" and "GHF-1 transcription factor"), syntactic variation, variation with abbreviations, and variation in enumeration (e.g., "TLR7/8" and "TLR7, TLR8") [20,21]. Orthologous genes usually belong to several species. To solve the GN task accurately, gene mentions must be assigned to the correct species and normalized to their own database identifiers. This is the most complicated step, and it arises from the extreme ambiguity of orthologous genes. For example, the ESR1 gene is associated with 22 Entrez Gene Ids, which belong to several different species. It is very difficult to normalize gene identifiers that lack species information. Intra-species gene ambiguity can occur when different genes have the same name. For example, the name "CAS" may refer to multiple distinct identifiers, such as Entrez Gene Id:1434 ("Cellular apoptosis susceptibility protein") or Entrez Gene Id:9564 ("Breast cancer antiestrogen resistance 1").

In this study, we propose an integrative method, GenNorm, to handle the three issues of the GN task. Our approach uses three modules, the gene name recognition (GNR) module, the species assignation (SA) module and the species-specific gene normalization (SGN) module. Good GNR processing can insure a high quality of gene mentions and reduce gene mention variation. SA is critical. Given a gene mention, it is essential to know to which species the gene belongs. Species information can help identify orthologous genes and help resolve the species discussed in each article. Ignoring the SA can lead to severe problems with gene ambiguity. SGN is the last and significant for GN; this module is responsible for intra-species gene ambiguity.

\section{Methods}

For the GN task, we developed an integration method consisting of three modules, as shown in Figure 1. The GNR module extracts gene mentions from full-text articles. This module applies a well-known system for 


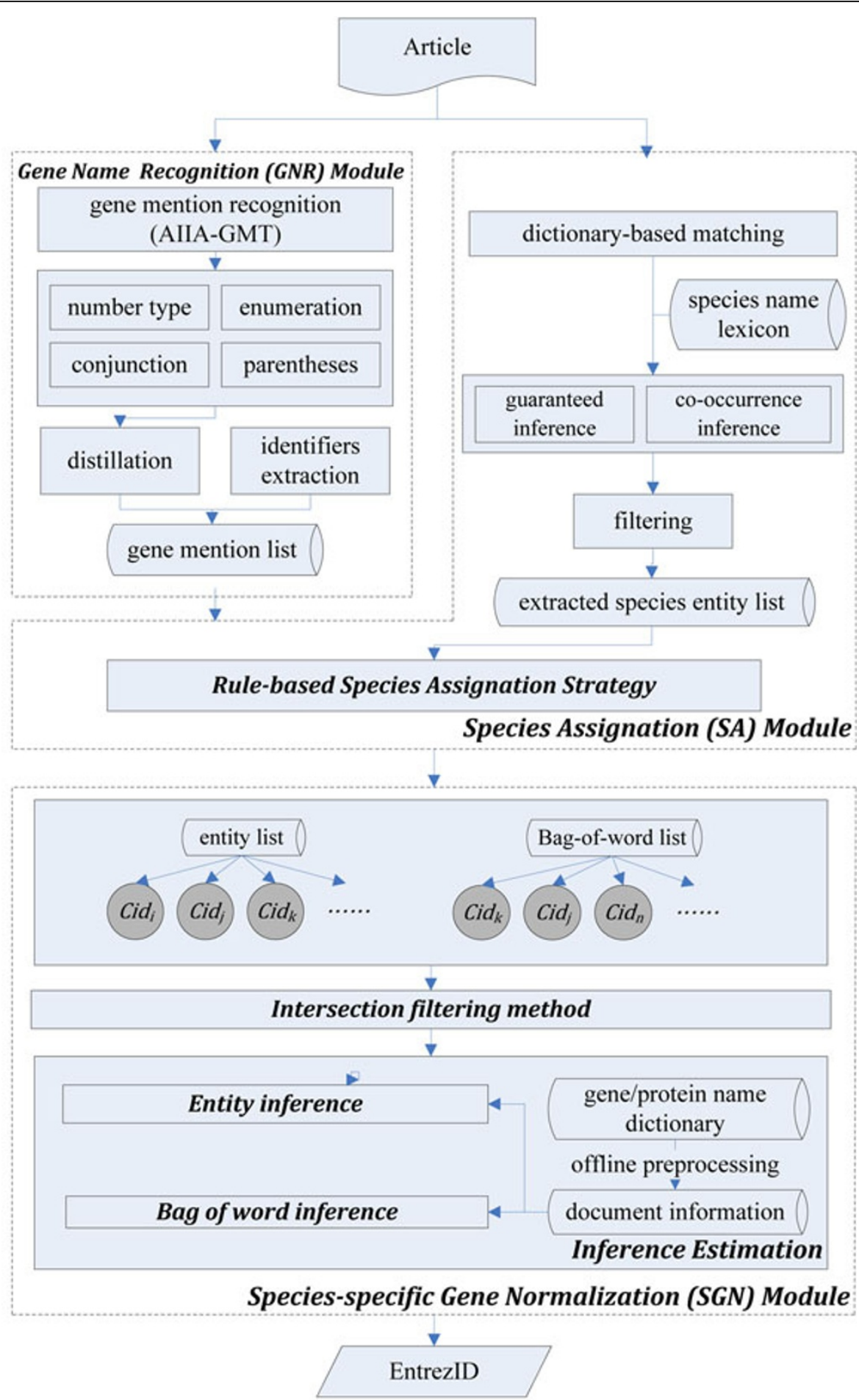

Figure 1 Architecture of GenNorm. GenNorm includes gene name recognition (GNR), species assignation (SA) and species-specific gene name normalization modules.

tagging gene mentions to tokenize them by proposed post-processing rules. The distillation strategy then filters non-gene names. The SA module applies a dictionary-based matching method with two robust inferring strategies to generate the species entity list. The species lexicon combines species and cell names (which can indicate their own species) to cover all kinds of species mentions. Then, the species assignation strategy uses 
contextual information to decrease the ambiguity of each gene mention. Finally, the SGN module uses an inference network model to handle intra-species gene ambiguity and gene-name variation.

\section{Gene name recognition module}

Our system uses the name-entity tagging tool AIIAGMT [22]. This tool is an XML-RPC client of a web server that recognizes named entities in biomedical articles. A general entity recognition process cannot collect sufficient information for entity normalization. These systems usually treat lightly or ignore informal, simplified naming descriptions, such as abbreviated names, enumeration mention descriptions, and names with conjunctions. Thus, we propose a post-processing step which can enhance the ability of general-purpose recognition systems.

Due to the varied naming styles of gene names in the biomedical literature, a tagged entity will not always exactly match a gene name in the dictionary. To address this issue, four translation rules of the post-processing step, i.e., the "number type", "conjunctions", "enumerations", and "parentheses", are applied to tokenize gene names (Table 1).

The first rule is the number type. Numbers of different subunit type, e.g., Roman, Arabic and Latin, are unified. Second, entities with conjunctions are split. Sometimes, two or more gene names have been combined into one mention by several conjunctions. This mention is split into several mentions. In the enumeration step, we extract the Arabic and Roman types by splitting conjunctions and combining the mutual family name with each Arabic or Roman type. At this step, an enumeration entity with the sequential numbers represents several gene names belonging to the same family. The entity is separated into several different gene names sharing their mutual family name. In the last rule, abbreviations in the parentheses of a gene entity are isolated. The "protein(s)" or "gene(s)" are then removed from the mentions, e.g., "MURF 3 protein" becomes "MURF 3".

After the post-processing, the method applies a distillation strategy to prevent false tagging. This step focuses on protein families, group and complex names, and non-gene terms from the tagged entities. In our performance observation in training data, filtering the protein families, group and complex names improved the precision, but the recall was lost. Therefore, the F-measure did not change, but the TAP-K score was improved. Thus, these equivocal terms are filtered in this strategy.

We apply four regular expressions (Table 2) to remove the filtering targets, which might include family names, figure names, or antibodies, for example. Some gene identifiers are mentioned in the articles, and these identifiers can be directly matched to their own species; however, most general taggers of gene mentions cannot extract identifiers mentions. For this particular situation, we attach an "identifier extraction" to the GNR module. To collect identifier mentions, we combine several kinds of gene identifiers, such as swissprot_id and SGD_id, from the locusTaganddbXrefs attributes of the gene_info table of the EntrezGene database and use the combined corpus to match identifier mentions. To reduce the computational cost, tokens with Arabic numerals or alphabetic characters are extracted from the articles. The extraction of candidate mention of identifiers is handled by two regular expressions: / $\backslash \mathrm{S}^{*}$ $\left.[0-9]+\backslash S^{*}[\mathrm{~A}-\mathrm{Za}-\mathrm{z}]+\backslash \mathrm{S}^{*}\right)\left(\left[{ }^{\wedge} 0-9 \mathrm{~A}-\mathrm{Za}-\mathrm{z}\right]+.^{* *}\right) \$ /$ and $/\left(\backslash \mathrm{S}^{*}[\mathrm{~A}-\right.$ $\left.Z a-z]+\backslash S^{*}[0-9]+\backslash S^{*}\right)([\wedge 0-9 A-Z a-z]+. *) \$ /$ The first matching by pair of parentheses is used to match the target tokens. The second pair of parentheses extracts the part of sentences after the matched target tokens. It is because that one sentence may contain more than one target token. Particular symbols, including "white", "hyphen", "dot" and "underline", are then removed from the tokens and gene identifiers before matching, e.g., "YCL057C-A" becomes "YCL057CA". After this adjustment, the tokens are compared with the gene identifiers. Tokens that exactly match gene identifiers are possible Entrez Gene Ids.

Lastly, the extracted entities are dumped into a "bag of words," as determined by their punctuation, symbols, and spaces. For example, "Hypoxia-inducible factor-1 alpha" is split into "Hypoxia", "inducible", "factor", "1" and "alpha", each of which is stored in the bag-of-words list.

Table 1 Several steps of post-processing of the GNR module.

\begin{tabular}{|c|c|c|}
\hline Step & Rules & Example \\
\hline Number type & $\begin{array}{l}\text { Roman } \rightarrow \text { Arabic Greek } \\
\rightarrow \text { English }\end{array}$ & I, II, III Roman $\rightarrow$ "1, 2, 3" Arabic alpha, beta, gamma $\rightarrow$ "a", "b", "g" \\
\hline Conjunctions & Split entity by and/or & $\begin{array}{l}\text { GABARAP and light chain } 3 \rightarrow \text { "GABARAP" \& "light chain } 3 \text { " furin or proprotein convertase } \rightarrow \text { "furin" and } \\
\text { "proprotein convertase" }\end{array}$ \\
\hline Enumerations & $\begin{array}{l}\text { Enumeration } \rightarrow \text { gene } \\
\text { mentions }\end{array}$ & Robo 1/2 $\rightarrow$ "Robo 1" and "Robo 2" SMADs 1, 5 and $8 \rightarrow$ "SMAD 1" and "SMAD 5" and "SMAD 8" \\
\hline Parentheses & $\begin{array}{l}\text { Split entity by } \\
\text { parentheses }\end{array}$ & $\begin{array}{l}\text { fibroblast growth factor-2 (FGF-2)-interacting-factor } \rightarrow \text { "FGF-2" and "fibroblast growth factor } 2 \text { interacting } \\
\text { factor" gamma carboxyglutamic acid (Gla) } \rightarrow \text { "gamma carboxyglutamic acid" and "Gla" }\end{array}$ \\
\hline
\end{tabular}


Table 2 Regular expressions used in the distillation strategy

\begin{tabular}{|c|c|}
\hline Filter set & Regular expression \\
\hline Family name & /([A-Za-Z0-9]+)[^A-Za-Z0-9](cell|family|subfamily|superfamily|domain|promotor)/ \\
\hline Attachment & /(fig|figure|video|movie|tab|table)[^A-Za-Z0-9]+([A-Za-Z0-9]+)/ \\
\hline Antibody name & $/($ anti) $[\wedge A-Z a-Z 0-9]+([A-Z a-Z 0-9]+)[\wedge A-Z a-Z 0-9]+($ antibody|antibodies $) /$ \\
\hline Biomedical words & $/([A-Z a-Z 0-9]+)[\wedge A-Z a-Z 0-9]+($ binding peptide|pathway|domain|cell|function|isoforms)/ \\
\hline
\end{tabular}

\section{Species assignation module}

To assign a suitable species to each gene mention, the first step is to extract species mentions from articles. We propose two robust inference strategies combined with a dictionary-based matching method. The species name collection aggregates three different species name lexicons: the NCBI taxonomy, list of cell lines from Wikipedia and the corpus of Linnaeus [9]. Sometimes, species do not map to any Entrez Gene Ids. For example, Escherichia of tax_id:561 does not map to any Entrez Gene Id. Such species are removed. The number of species in the original lexicons is 570,679; after filtering, the number species in the combined lexicon is 6,764.

Synonyms of every species in the lexicon are used to detect species names using dictionary-based matching. A species may have a variety of abbreviation names, e.g., "Escherichia coli strain k-12" is same as "E.coli k-12" and "E. coli k-12". Some species have an especially large number of synonyms. To handle this case, a dictionary extension strategy is used. We automatically generate additional synonyms from each species name by replacing the genus name with its first letter and a dot and potentially a white space [10], e.g., "E. coli”. These species synonyms are then added if they do not already occur in the collection of lexicons. All uppercase letters are then changed to a lowercase form. For example, Taxonomy ID (tax_id): 83333 contains several synonyms, including "escherichia coli k-12", "escherichia coli k12", "e.coli k-12" and "e. coli k-12".

Checking all synonyms of a species is a time-consuming task. To enhance the computation speed, all synonymous names of a species are integrated and transformed into a regular expression automatically. In other words, the regular expression of a tax_id contains all existing names of the species. For the given example, tax_id:83333 would lead to the expression "e(?:^. ?coli k ।-12|scherichia coli $\mathrm{k} \backslash$-?12)".

The NCBI Taxonomy is a hierarchical structure of species types. Our collection only includes four types, "species", "no rank", "subspecies" and "variants". Additionally, we add the genus, taken from the first word of each species' scientific name, to the species dictionary. These genus names will be used to infer the correct tax_id, as will be discussed below. (We set the priorities for the disambiguation of species names as "species" > "no rank" > "subspecies" > "variants" > "genus name", with the species type on the left a higher priority than the type on the right). When two species have the same name, the lower priority one is eliminated.

There are some additional cases of non-matching results. First, some species entities are genus names. These entities in the same article always indicate a mutual species of the genus, e.g., "Arabidopsis" is recognized as "Arabidopsis thaliana" when the same article includes both entities. Second, family species will share the same species name. An example is shown in Table 3 for the ambiguous Escherichia coli species family, which includes 45 taxonomy identifiers, such as 511145, 431946, and 511693. For tax_id:511145, the last subtype of the species is "mg1655", and it can indicate the species mention "Escherichia coli" to tax_id:511145.

To handle these cases, we devised two robust strategies of robust inference. (1) Guaranteed inference: guaranteed entities can be used to disambiguate unguaranteed entities. The complete species name is guaranteed to indicate tax_id, but genus names, ambiguous cell names and abbreviations are unguaranteed. The substitution of genus for species name can also be described as a type of anaphora. Unguaranteed entities always occur with the guaranteed name in articles, e.g., "Arabidopsis" accompanies "Arabidopsis thaliana" and “A549” accompanies "A549 cell(s)”. In reverse, "A549” cannot imply "A549 cell" when the article does not contain the complete species name "A549 cell(s)". The guaranteed entity can imply the unguaranteed entities that indicate the same species. As an example (Figure 2),

\section{Table 3 Species family of Escherichia coli (tax_id:562) including 45 species.}

\begin{tabular}{ll}
\hline tax id & Species scientific name \\
\hline 199310 & escherichia coli cft073 \\
316385 & escherichia coli str. k-12 substr. dh10b \\
316401 & escherichia coli etec h10407 \\
331111 & escherichia coli e24377a \\
331112 & escherichia coli hs \\
344610 & escherichia coli 53638 \\
413997 & escherichia coli b str. rel606 \\
431946 & escherichia coli se15 \\
83333 & escherichia coli k-12 \\
511145 & escherichia coli str. k-12 substr. mg1655 \\
$\ldots$ & $\ldots$
\end{tabular}




\section{Articles ID:288058}

In order to further investigate the role of NOX2 in virus-mediated IRF- 3 activation, we first thought to confirm, in our A549 model, the essential role of RIG$\mathrm{I}$ in $\mathrm{SeV}$ recognition that was previously highlighted in embryonic fibroblasts

(MEFs), ... demonstrating that RIG-I is essential for downstream signaling to IRF-3 in the early time points following $\mathrm{SeV}$ infection in $\mathbf{A 5 4 9}$ cells.

Figure 2 Example of guaranteed inference. The unguaranteed species name-"A549" can be inferred to human species by guaranteed species name-"A549 cells".

"A549 cells" can imply that "A549" indicates an A549 cell. Thus, the "A549 cell" mention frequency is two in this paragraph. (2) Co-occurrence inference: the species sub-type can disambiguate the species name and genus name, when the sub-type appears after the species and genus names in the same sentence. The sub-types in the NCBI Taxonomy include strain, substrain, variant, subspecies, pathovars and biovar. For example, "MG1655" is a substrain of "E. coli K12".

Figure 3 shows how "E. coli K12 (MG1655)" appears different from all synonyms of tax_id:511145. Dictionary-based matching is not useful in this situation. In our observation, each subtype can be unambiguously assigned to one species. Thus, the MG1655 can disambiguate "Escherichia", "E. coli" and "E. coli K12" to the specific species (tax_id:511145).

We show in Table 4 the types of species mentions by species extracting step in species assignation module. The targets of guaranteed inference are "genus name" and "cell line", and the target of co-occurrence inference is "sub-type". The total percentage of inference targets among species mentions is $11.3 \%(8.06 \%+2.04 \%+1.20 \%)$. However, these targets are not a major part of species mentions. It is nonetheless the most difficult aspect of detecting species mentions. Lastly, a set of high-falsepositive aggregated from species mentions is shown in Table 5 . The first row is collected by Linnaeus software [9]. Candidate species terms matching high false positive terms are removed. Two experiment descriptors in the second row contain "yeast"; similar species names would lead to false positives, and the "yeast" in these two experiment terms is removed. In addition, particular tagged entities are shortened, e.g., the prefixes or suffixes of antibody-related terms, as shown in the third row. For example, the species mentions "flag", "goat"

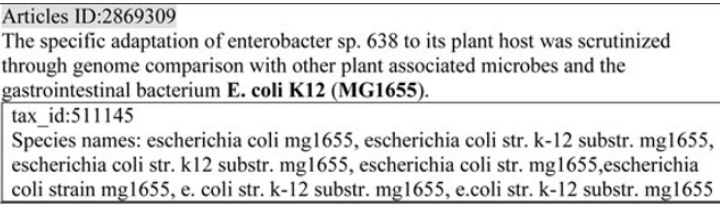

The specific adaptation of enterobacter sp. 638 to its plant host was scrutinize through genome comparison with other plant associated microbes and the gastrointestinal bacterium E. coli K12 (MG1655).

tax id:511145

Species names: escherichia coli mg1655, escherichia coli str. k-12 substr. mg1655, escherichia coli str. k12 substr. mg1655, escherichia coli str. mg1655, escherichia coli strain mg1655, e. coli str. k-12 substr. mg1655, e.coli str. k-12 substr. mg1655

Figure 3 Example of co-occurrence inference. The substrain"MG1655" can infer the species name-"E. coli K12" to "E. coli str. k-12 substr. mg1655" (tax_id:511145). and "mouse" in "anti-flag antibody", "goat anti-Rad53 polyclonal antibody" and "anti-HA9 mouse monoclonal antibody" are filtered.

There are 5,933,419 Entrez Gene Ids belonging to more than 6,000 species. Genes are ambiguous among the other genes of the same species and among orthologs. Therefore, we developed a rule-based species assignation strategy to reduce orthologous gene ambiguity.

After the species is extracted, each gene entity is assigned the suitable tax_id. Based on Wang et al. [8], we defined several species indicators of tax_id assignment for gene mentions. Details are shown in Table 6 . Boldface terms represent species names, and underlined words are gene mentions.

Table 7 shows an example using the frequency of species mentions from PMCID: 2880583. The tax_id:9606 (Human) is used most frequently in this article, and there is 1 instance of "293 cell", 34 instances of "a549 cell" and 6 instances of "human". If there are no other species mentions in the article, all gene mentions are assigned by tax_id: 9606 (human).

\section{Species-specific gene normalization module}

After species assignation, the proposed SGN module, based on previous work [23], measures the inference scores of candidate Entrez Gene Ids in articles. The previous study applied an inference network model to the GN task, but it did not handle orthologous gene ambiguity. We applied the inference network model to collect the exact match and partial match between tagged entities from articles and gene name entities from the gene dictionary. The inference by exact match is named entity inference, and the inference by partial match is named bag of word inference. Exact match means that tagged entity and gene name entity must be the same term, and partial match means that at least one word in the bags of words of a tagged entity and of a gene name entity must be the same word. The model applied entities and bag of words to the same estimation. The original model gave equal weight to entities and bags of words, thus could not distinguish the relative importance of entities and words from the bags of words. This design disadvantaged the GN performance. We reorganized the previous design by splitting the inference network model into two inference estimations.

In SGN, entity inference and bag-of-words inference are used to measure confidence scores. The gene name entities are divided into two lists, an entity list and a bag-of-words list. The entity list stores the output of the GNR module, and the bag of words list contains all bags of words from the entity list. Each record of these two lists is used to obtain a candidate Entrez Gene Id ( $\mathrm{Cid}$ ). Before inference estimations, the Cid is filtered by the intersection filtering method described below. The two 
Table 4 Types of species mentions extracted by two Inferring types.

\begin{tabular}{llll}
\hline Species type & Inferring type & Number of species mentions & Ratio \\
\hline Genus name & Guaranteed-inference & 2,824 & $8.06 \%$ \\
Cell line & Guaranteed-inference & 715 & $2.04 \%$ \\
Sub-type & Co-occurrence inference & 422 & $1.20 \%$ \\
General species name & - & 31,093 & $88.70 \%$ \\
Total & - & 35,054 & $100.00 \%$ \\
\hline
\end{tabular}

The total frequency of inference targets among species mentions is $11.3 \%$.

inference estimations apply a TF-IDF-based inference network to determine the possible Entrez Gene Ids for each article. Consider the inference example in Figure 4, Entrez Gene Id: 51554 includes entities "CCRL", "CCRL1", "chemokine receptor like 1" and "orphan seven-transmembrane receptor", etc. PMID: 10767544 consists of entities "CCRL1", "chemokine receptor like 1 " and "orphan seven transmembrane receptor related to chemokine receptors", etc. Those entities are used to construct entity inference and bag-of-word inference estimations. The entity inference is constructed by the exact match between gene name entities from Entrez Gene Id: 51554 and tagged entities from PMID: 10767544. There are two exact matches in entity inference, e.g. "CCRL1" and "chemokine receptor like 1". Then, the bag-of-word inference is constructed by partial match between bags of words of gene name entities from Entrez Gene Id: 51554 and tagged entities from PMID: 10767544, such as "chemokine", "receptor" and "like".

For disambiguating confused Cids and decreasing the computation cost, we discard many irrelevant Cids by our intersection filtering method. All pairs of Cids are compared: all terms extracted by exact and partial match are considered in each Cid. A Cid is removed when its term list is a subset of the term list of another Cid.

\section{Results}

Our system was run on the evaluation data of the BioCreative III GN [24] task training and test corpora, as shown in Table 8 . The test data were unknown to our system until the official runs were executed. The training set included two sets of annotated full-length articles. The first set had been fully annotated by a group of trained and experienced curators, who had been invited from various model organism databases. The second set was partially annotated: only the most important genes had been annotated by human indexers at the National Library of Medicine. The test set included 507 full text articles from various BMC and PLoS journals. To understand the differences in the results between teams, organizers selected the 50 articles that presented the most difficult and varied results to evaluate the submissions of the teams. The "gold standard" of the 50 selected articles was annotated manually, and the "silver standard" of 507 articles including the 50 "gold standard" articles was generated automatically by an Expectation Maximization (EM) algorithm using the best submissions of all teams. The numbers of gene IDs common to the shared 50 gold- and silver-standard articles is just 528. The annotated gene identifiers of the gold standard are very dissimilar to silver standard.

Carrol et al. [25] proposed a new metric, threshold average precision (TAP- $k$ ), for measuring retrieval efficacy of GN task performance. In short, TAP-k is the mean average precision with a variable cutoff and a terminal cutoff penalty. Evaluations using the gold and silver standard annotations are shown in Table 9; note that the set of 50 gold standard articles is a part of the 507 silver standard articles. We obtained the highest TAP-k scores on the gold standard: $0.3297(\mathrm{k}=5), 0.3538$ $(\mathrm{k}=10)$, and $0.3535(\mathrm{k}=20)$. In silver-standard-507 evaluation, our TAP-k scores are 0.4591 for $\mathrm{k}=5,10$, and 20 . Our system was ranked $2^{\text {nd }}, 2^{\text {nd }}$, and $3^{\text {rd }}$ in terms of TAP-5, TAP-10, and TAP-20 respectively on the 507 full-text test articles.

The annotation of the silver standard depends on the submissions of all teams. Because the best submission might be dissimilar to other teams' submissions, its relative performance on the silver standard might suffer. Nevertheless, the two best runs with the gold standard from our submission are still among the ones with the highest TAP-k score with the silver standard. In addition,

Table 5 High-false-positive set.

\begin{tabular}{ll}
\hline Filter set & Terms \\
\hline High-false-positive terms & 3a (215167), t7 (10760), cat (9685), ass (9793), j1 (1829) \\
Experiment terms & yeast two hybrid (4932), yeast 2 hybrid (4932) \\
Antibody & anti-, antibodies, polyclonal, igg, serum, monoclonal, antibody \\
\hline
\end{tabular}


Table 6 Species indicators of species assignation strategy which are adapted from Wang et al. [8]

\begin{tabular}{|c|c|c|}
\hline $\begin{array}{l}\text { Species } \\
\text { indicators }\end{array}$ & Description & Example \\
\hline Identifier & $\begin{array}{l}\text { The gene is assigned a species when the gene is extracted by } \\
\text { gene identifier extraction. }\end{array}$ & $\begin{array}{l}\text { A bar for Cat8 is not included, as only one gene (YOR019W, factor } \\
\text { change 2.1) has a Cat8 site alone. }\end{array}$ \\
\hline Prefix & $\begin{array}{l}\text { The first lowercase letter of the gene name is an abbreviation of } \\
\text { its species. }\end{array}$ & hZIP 2 (human gene) \\
\hline $\begin{array}{l}\text { Previous } \\
\text { indicator }\end{array}$ & $\begin{array}{l}\text { The tax_id is assigned to a gene entity if the species entity } \\
\text { appears in front of the gene entity. }\end{array}$ & $\begin{array}{l}\text { DrosophilaHCF } \\
\text { zebraflshLtk }\end{array}$ \\
\hline $\begin{array}{l}\text { Forward } \\
\text { indicator }\end{array}$ & $\begin{array}{l}\text { The tax_id is assigned to a gene entity if the species entity is in } \\
\text { front of the gene entity in the same sentence. The nearest species } \\
\text { is used for assignment. }\end{array}$ & $\begin{array}{l}\text { Conversely, ISWI is a unique and essential gene in Drosophila, } \\
\text { highlighting a possible divergent role for ISWI in flies and a distinct } \\
\text { mechanism of interaction with the Sin3A/Rpd3 complex in higher } \\
\text { eukaryotes. }\end{array}$ \\
\hline $\begin{array}{l}\text { Backward } \\
\text { indicator }\end{array}$ & $\begin{array}{l}\text { The tax_id is assigned to a gene entity if the species entity } \\
\text { precedes the gene entity in the same sentence. The nearest } \\
\text { species is used for assignment. }\end{array}$ & $\begin{array}{l}\text { We identify the shady gene as encoding a cell signaling receptor, } \\
\text { leukocyte tyrosine kinase } \\
\text { with human auto-immune disease. }\end{array}$ \\
\hline $\begin{array}{l}\text { Majority } \\
\text { indicator }\end{array}$ & $\begin{array}{l}\text { The most frequently mentioned tax_id is assigned to the gene } \\
\text { entity if it cannot be assigned by previous rules. }\end{array}$ & $\begin{array}{l}\text { The most frequent mention of PMCID: } 2880583 \text { is tax_id is } 9606 \\
\text { (Human) as shown in Table } 7 .\end{array}$ \\
\hline
\end{tabular}

the four teams (numbers 83, 74, 98 and 101) that performed best on the gold standard consistently remained in the top tier in silver-standard evaluations. It is evident that relative rankings tended to be preserved in this comparison. Evaluation with the silver-standard annotation proves that the automatic annotation works [24].

In addition, we calculated precision, recall and F-measure to evaluate the accuracy and coverage of our result (Team 83). We obtained an F-measure of $46.56 \%$ with the gold standard, $46.90 \%$ with the silver standard with 50 articles and 55.09\% with the silver standard with 507 articles (Table 10).

To better understand the contribution of each component in the GN method, we sequentially ran the system over the test data without each component of each module. Table 11 shows how each component contributed to GN performance. The first row shows the TAP-k $(k=5,10,20)$ score when all components were used. The other rows show the performance when one of the components was missing. The values in parentheses are the decrement of the component removals.

\section{Discussion}

In the following section, we first discuss the impact of the GNR module in the context of three extension components of the gene mention recognition system (AIIA-

Table 7 An example of the frequency of species mentions by PMCID: 2880583.

\begin{tabular}{llcc}
\hline PMCID & Species and cell name & tax id & Num \\
\hline 2880583 & 293(1), a549(34), human(6) & 9606 & 41 \\
& bovine(1) & 9913 & 1 \\
& mice(1), murine(1) & 10090 & 2 \\
& rabbit(1) & 9986 & 1 \\
& sendai virus(2) & 11191 & 2 \\
\hline
\end{tabular}

GMT). The impact of two robust referring strategies, the filtering processing and five assignation rules (because identifier extraction is repeated in the GNR module, we do not perform the same experiment again) of SA module are described. Finally, we analyze the performance of the SGN module by the intersection filtering method and inference network.

First, we found that the post-processing step is a useful component of the GNR module. If the output of AIIA-GMT were not followed by post-processing, performance would clearly decrease. The most effective component is identifier extraction; several popular and high-performance tagging systems, such as AIIA-GMT [22], ABNER [26], and GENIA Tagger [27], usually cannot recognize gene identifiers well.

The three rows under the SA module (Table 11) show the performance when one of the two robust inference strategies, the filtering processing and six species assignation rules, was unused. The disambiguation of species mentions is critical, which is why we designed two robust referring strategies to handle it. Using only one of the two robust referring strategies caused a decline in the performance of the species assignation rules and an approximately $6 \%-7 \%$ decrease in TAP-k scores. The effect of the two robust inference strategies is not obvious; the ratio of species mentions that are unguaranteed species names (i.e., genus and cell line) and subtype is just $11.3 \%$ (Table 4 ). The filtering of false-positive set is very useful. The next most helpful indicators of species assignation are "Forward indicator" and "Majority indicator". Especially for "Majority indicator", the detection of the species covered in the article is an important issue $[8,10]$. Majority indicator is most popular. Furthermore, the precise extraction of species mentions leads to good performance of the Majority indicator. 


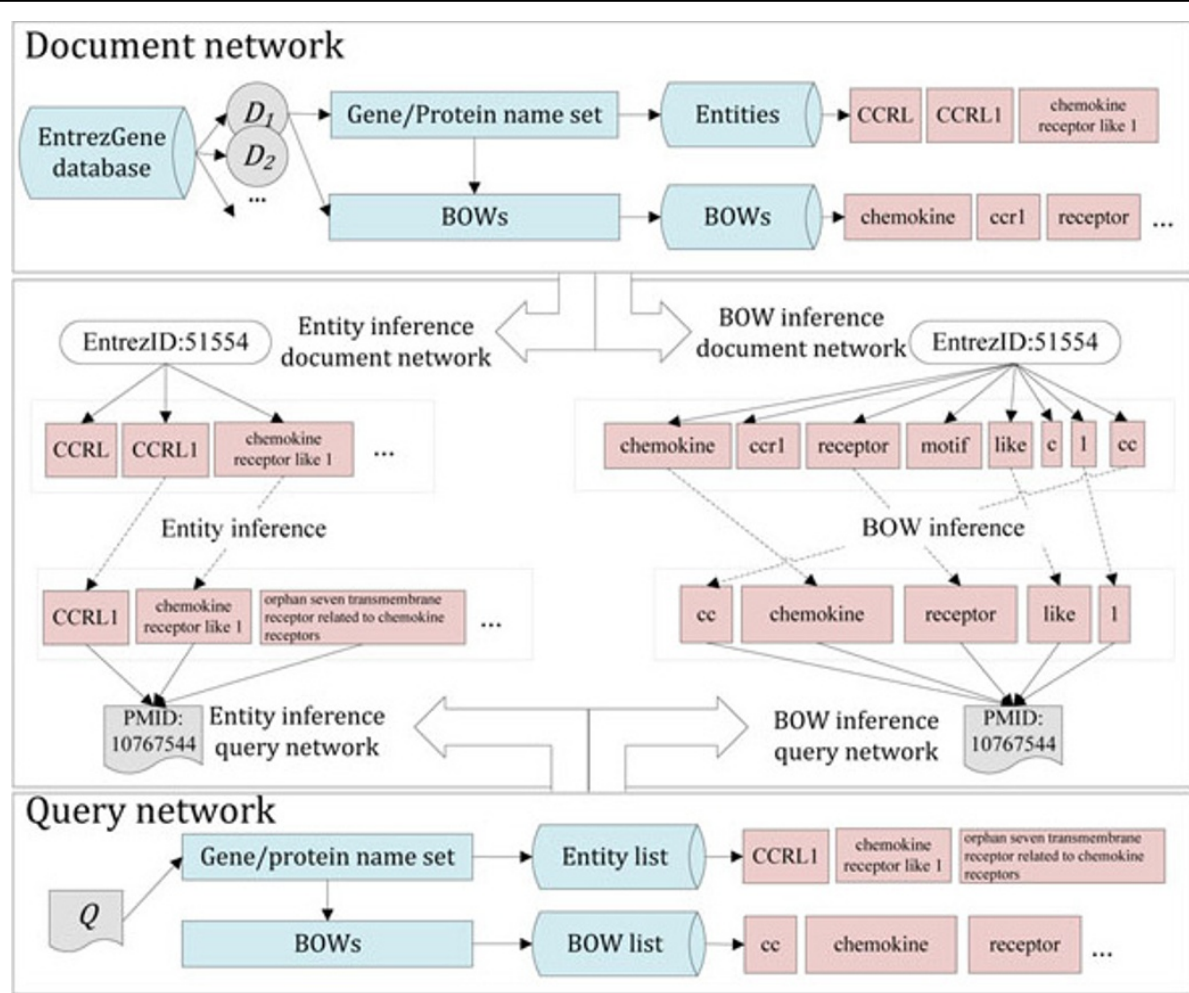

Figure 4 Example of species-specific gene normalization by two inference estimations including entity and BOW inference.

Table 8 Statistics of annotations of full-text articles

\begin{tabular}{llllll}
\hline & Training (fully annotated) & Training (partially annotated) & Test (gold standard) & Test (silver standard) \\
\cline { 2 - 5 } & & & 50 & 507 & 50 \\
\hline Total number of full-text articles & 32 & 525 & 1669 & 507 & 50 \\
Total gene IDs of each annotation & 607 & 770 & 33.32 & 9378 & 1828 \\
Avg. gene IDs of each annotation & 18.97 & 1.467 & 18.50 & 36.56 \\
\hline
\end{tabular}

Table 9 Performance on the gene normalization task by the top 4 performing teams in the BioCreative III competition

\begin{tabular}{|c|c|c|c|c|c|c|c|c|c|c|}
\hline \multirow[t]{2}{*}{ Teams } & & \multicolumn{3}{|c|}{ Gold standard (50 selected articles) } & \multicolumn{3}{|c|}{ Silver standard (50 selected articles) } & \multicolumn{3}{|c|}{ Silver standard (All 507 articles) } \\
\hline & & TAP-5 & TAP-10 & TAP-20 & TAP-5 & TAP-10 & TAP-20 & TAP-5 & TAP-10 & TAP-20 \\
\hline \multirow[t]{3}{*}{ Kuo et al. (Team 74) } & 1st run & 0.2137 & 0.2509 & 0.2509 & 0.3820 & 0.3820 & 0.3820 & 0.4873 & 0.4873 & 0.4873 \\
\hline & 2nd run & 0.2083 & 0.2480 & 0.2480 & 0.3855 & 0.3855 & 0.3855 & 0.4871 & 0.4871 & 0.4871 \\
\hline & 3rd run & 0.2099 & 0.2495 & 0.2495 & 0.3890 & 0.3890 & 0.3890 & 0.4916 & 0.4916 & 0.4916 \\
\hline \multirow[t]{3}{*}{ Our method (Team 83) } & 1st run & 0.3254 & 0.3538 & 0.3535 & 0.3567 & 0.3600 & 0.3600 & 0.4591 & 0.4591 & 0.4591 \\
\hline & 2nd run & 0.3216 & 0.3435 & 0.3435 & 0.3291 & 0.3291 & 0.3291 & 0.4323 & 0.4323 & 0.4323 \\
\hline & 3rd run & 0.3297 & 0.3514 & 0.3514 & 0.3382 & 0.3382 & 0.3382 & 0.4327 & 0.4327 & 0.4327 \\
\hline \multirow[t]{3}{*}{ Liu et al. (Team 98) } & 1st run & 0.2835 & 0.3012 & 0.3103 & 0.3343 & 0.3535 & 0.3629 & 0.3818 & 0.3899 & 0.3875 \\
\hline & 2nd run & 0.2909 & 0.3079 & 0.3087 & 0.3354 & 0.3543 & 0.3634 & 0.3790 & 0.3878 & 0.3868 \\
\hline & 3rd run & 0.3013 & 0.3183 & 0.3303 & 0.3710 & 0.4116 & 0.4672 & 0.4086 & 0.4511 & 0.4648 \\
\hline \multirow[t]{3}{*}{ Lai et al. (Team 101) } & 1st run & 0.1896 & 0.2288 & 0.2385 & 0.3590 & 0.3859 & 0.3859 & 0.4289 & 0.4289 & 0.4289 \\
\hline & 2nd run & 0.1672 & 0.2150 & 0.2418 & 0.3239 & 0.3945 & 0.4132 & 0.4294 & 0.4408 & 0.4408 \\
\hline & 3rd run & 0.1812 & 0.2141 & 0.2425 & 0.3258 & 0.4109 & 0.4109 & 0.4536 & 0.4536 & 0.4536 \\
\hline
\end{tabular}


Table 10 Performance statistics evaluated by TAP-K and F-measure on test data and training data sets.

\begin{tabular}{llllllll}
\hline Corpus & Data set & TAP-5 & TAP-10 & TAP-20 & Precision & Recall & F-measure \\
\hline Test data (1st run) & 50 (gold standard) & 0.3254 & $\mathbf{0 . 3 5 3 8}$ & $\mathbf{0 . 3 5 3 5}$ & $53.85 \%$ & $39.44 \%$ & $45.53 \%$ \\
Test data (2nd run) & 50 (gold standard) & 0.3216 & 0.3435 & 0.3435 & $55.54 \%$ & $39.07 \%$ & $\mathbf{4 5 . 8 7 \%}$ \\
Test data (3rd run) & 50 (gold standard) & $\mathbf{0 . 3 2 9 7}$ & 0.3514 & 0.3514 & $\mathbf{5 6 . 2 3 \%}$ & $\mathbf{3 9 . 7 2 \%}$ & $\mathbf{4 6 . 5 6 \%}$ \\
\hline Test data (1st run) & 50 (silver standard) & $\mathbf{0 . 3 5 6 7}$ & $\mathbf{0 . 3 6 0 0}$ & $\mathbf{0 . 3 6 0 0}$ & $58.94 \%$ & $\mathbf{3 8 . 9 5 \%}$ & $\mathbf{4 6 . 9 0 \%}$ \\
Test data (2nd run) & 50 (silver standard) & 0.3291 & 0.3291 & 0.3291 & $58.60 \%$ & $37.64 \%$ & $\mathbf{4 5 . 8 4 \%}$ \\
Test data (3rd run) & 50 (silver standard) & 0.3382 & 0.3382 & 0.3382 & $\mathbf{5 9 . 4 6 \%}$ & $38.35 \%$ & $\mathbf{4 6 . 6 2 \%}$ \\
\hline Test data (1st run) & 507 (silver standard) & $\mathbf{0 . 4 5 9 1}$ & $\mathbf{0 . 4 5 9 1}$ & $\mathbf{0 . 4 5 9 1}$ & $71.79 \%$ & $\mathbf{4 4 . 6 9 \%}$ & $\mathbf{5 5 . 0 9 \%}$ \\
Test data (2nd run) & 507 (silver standard) & 0.4323 & 0.4323 & 0.4323 & $72.08 \%$ & $42.70 \%$ & $53.64 \%$ \\
Test data (3rd run) & 507 (silver standard) & 0.4327 & 0.4327 & 0.4327 & $\mathbf{7 2 . 4 1 \%}$ & $42.82 \%$ & $53.82 \%$ \\
\hline Training data & 32 (gold standard) & 0.4703 & 0.4969 & 0.4969 & $63.82 \%$ & $67.71 \%$ & $65.70 \%$ \\
\hline
\end{tabular}

Finally, the SGN module includes two major parts: intersection filtering and inference estimation. The contribution of the intersection filtering method is to enhance computing speed, and thus the effect of this method on performance is not remarkable. To evaluate the contribution of the inference network model of two estimations, we replaced this model by a simple vector space model. The performance decreased substantially.

We evaluated our method using the full-text articles provided in the BioCreative III competition. Our best result had TAP-k scores of $0.3297 \quad(k=5), 0.3538 \quad(k=10)$, and $0.3535(\mathrm{k}=20)$ under the gold-standard evaluation.

Our method approaches the challenges of GN as a series of tasks, with several issues handled by respective modules. Thedisadvantageousdesigns of each module may lead to a decline in overall performance. The major goal of this work is to present the architecture of our method in a clear way and analyze the effective components, which we do through systematic removals. This analysis can help in the redesigning of each segment to create a better system.

\section{Conclusions}

The GN task of BioCreative III was more difficult than previous GN tasks, and the chief reason is orthologous gene ambiguity. In this study, we focused on the issue of gene normalization in species assignation and developed an integrated method for mapping a biomedical entity to the correct Entrez Gene Id. To obtain good performance, we focused on ameliorating the effects of gene mention variation, orthologous gene ambiguity and intra-species gene ambiguity. The integrated method consists of three modules, GNR, SA and SGN, which function serially to handle these three issues. We participated in the GN task of the BioCreative III competition by adopting an integrated method based on our previous work to handle intra-species gene ambiguity. Results demonstrated that our method worked well,

Table 11 Contribution of each component of modules

\begin{tabular}{llll}
\hline Remove component & TAP-5 & TAP-10 & TAP-20 \\
\hline None & 0.3254 & 0.3538 & 0.3535 \\
GNR module & & & $0.3063(-13.35 \%)$ \\
Post-processing of AllA-GMT [22] & $0.2653(-18.47 \%)$ & $0.3063(-13.43 \%)$ & $0.3355(-5.09 \%)$ \\
Distillation strategy & $0.3094(-4.92 \%)$ & $0.3355(-5.17 \%)$ & $0.2888(-18.30 \%)$ \\
Identifier extraction & $0.2706(-16.84 \%)$ & $0.2888(-18.37 \%)$ & $0.3319(-6.11 \%)$ \\
SA module & & & $0.2644(-25.21 \%)$ \\
Two robust inference strategies & $0.3014(-7.38 \%)$ & $0.3319(-6.19 \%)$ & $0.3383(-4.30 \%)$ \\
Identifier & $0.2538(-22.00 \%)$ & $0.2538(-28.26 \%)$ & $0.3361(-4.92 \%)$ \\
Prefix & $0.3119(-4.15 \%)$ & $0.3383(-4.38 \%)$ & $0.3056(-13.55 \%)$ \\
Previous indicator & $0.3099(-4.76 \%)$ & $0.3361(-5.00 \%)$ & $0.3330(-5.80 \%)$ \\
Forward indicator & $0.2846(-12.54 \%)$ & $0.3056(-13.62 \%)$ & $0.2280(-35.50 \%)$ \\
Backward indicator & $0.3056(-6.08 \%)$ & $0.3330(-5.88 \%)$ & $0.2280(-35.56 \%)$ \\
Majority indicator & $0.2280(-29.93 \%)$ & & $0.3039(-14.03 \%)$ \\
SGN module & & $0.3411(-3.59 \%)$ & $0.3039(-14.10 \%)$ \\
Intersection filtering method & $0.3121(-4.09 \%)$ & &
\end{tabular}


ranking at the top level of performance among all teams. Our proposed method makes sufficient use of gene/species information in context and of a thesaurus of gene/ species.

Nonetheless, the current, state-of-the-art performance on the GN task is not good enough. The mining of fulltext articles and cross-species normalization are big challenges for GN. To improve future performance, the contexts of articles will be used, e.g., chromosomal locations, families, functions.

\section{Acknowledgements}

This research was partially supported by BioCreativelll workshop and CNIO institute. Authors would like to thank Zhiyong Lu at BioCreative GN task for his patience in responding to myriad questions about the evaluation. They would also like to think Chun-Nan Hsu who provided AllA-GMT system for GNR.

This article has been published as part of BMC Bioinformatics Volume 12 Supplement 8, 2011: The Third BioCreative - Critical Assessment of Information Extraction in Biology Challenge. The full contents of the supplement are available online at http://www.biomedcentral.com/1471$2105 / 12$ ? issue $=$ S8

\section{Competing interests}

The authors declare that they have no competing interests.

Published: 3 October 2011

\section{References}

1. Alex B, Grover C, Haddow B, Kabadjov M, Klein E, Matthews M, Roebuck S, Tobin $R$, Wang $X$ : Assisted curation: does text mining really help? Pac Symp Biocomput 2008, 556-567.

2. Hakenberg J, Plake C, Leaman R, Schroeder M, Gonzalez G: Inter-species normalization of gene mentions with GNAT. Bioinformatics 2008 24(ECCB):i126-i132.

3. Heinz JF, Mevissen T, Dach H, Oster M, Hofmann-Apitius M: ProMiner: Recognition of Human Gene and Protein Names using regularly updated Dictionaries. the Second BioCreative Challenge Evaluation Workshop 2007, 149-151

4. Hirschman L, Colosimo M, Morgan A, Yeh A: Overview of BioCreAtlvE task 1B: normalized gene lists. BMC Bioinformatics 2005, 6(Suppl 1):S11.

5. Leitner F, Mardis SA, Krallinger M, Cesareni G, Hirschman LA, Valencia A: An Overview of BioCreative II.5. IEEE/ACM Transactions On Computational Biology And Bioinformatics 2010, 7(3):385-399.

6. Morgan AA, Lu Z, Wang X, Cohen AM, Fluck J, Ruch P, Divoli A, Fundel K, Leaman R, Hakenberg J, et al: Overview of BioCreative II gene normalization. Genome Biology 2008, 9(Suppl 2):S3.

7. Wermter J, Tomanek K, Hahn U: High-Performance Gene Name Normalization with GENO. Bioinformatics 2009.

8. Wang X, Tsujii Ji, Ananiadou S: Disambiguating the Species of Biomedical Named Entities using Natural Language Parsers. Bioinformatics 2010 26(5):661-667.

9. Gerner M, Nenadic G, Bergman CM: LINNAEUS: A species name identification system for biomedical literature. BMC Bioinformatics 2010, $11(85)$.

10. Kappeler T, Kaljurand K, Rinaldi F: TX Task:Automatic Detection of Focus Organisms in Biomedical Publications. Proceedings of the Workshop on BioNLP: 2009 2009, 80-88.

11. Klinger R, Kolarik C, Fluck J, Hofmann-Apitius M, Friedrich CM: Detection of IUPAC and IUPAC-like chemical names. Bioinformatics 2008, 24(ISMB2008): i268-i276.

12. Corbett P, Batchelor C, Teufel S: Annotation of Chemical Named Entities, BioNLP 2007: Biological, translational, and clinical language processing 2007, 57-64.

13. Hakenberg J, Plake C, Royer L, Strobelt H, Leser U, Schroeder M: Gene mention normalization and interaction extraction with context models and sentence motifs. Genome Biology 2008, 9(Suppl 2):S14.
14. Hakenberg J, Leaman R, Vo NH, Jonnalagadda S, Miller RSC, Tari L, Baral C Gonzalez G: Efficient Extraction of Protein-Protein Interactions from FullText Articles. IEEE/ACM Transactions On Computational Biology And Bioinformatics 2010, 7(3):481-494.

15. Chen Y, Liu F, Manderick B: BioLMiner System: Interaction Normalization Task and Interaction Pair Task in the BioCreative II.5 Challenge. IEEE/ACM Transactions On Computational Biology And Bioinformatics 2010, 7(3):428-441.

16. Verspoor K, Roeder C, Johnson HL, Cohen KB Jr., W AB, Hunter LE: Exploring Species-Based Strategies for Gene Normalization. IEEE/ACM Transactions On Computational Biology And Bioinformatics 2010, 7(3):462-471

17. Saetre R, Yoshida K, Miwa M, Matsuzaki T, Kano Y, Tsujii Ji: Extracting Protein Interactions from Text with the Unified AkaneRE Event Extraction System. IEEE/ACM Transactions On Computational Biology And Bioinformatics 2010, 7(3):442-453.

18. Dai HJ, Lai PT, Tsai RTH: Multistage Gene Normalization and SVM-Based Ranking for Protein Interactor Extraction in Full-Text Articles. IEEE/ACM Transactions On Computational Biology And Bioinformatics 2010, 7(3):412-420.

19. Maglott D, Ostell J, Pruitt KD, Tatusova T: Entrez Gene: gene-centered information at NCBI. Nucleic acids research 2006, 00(Database issue):D1-D6

20. Hirschman L, Morgan AA, Yeh AS: Rutabaga by any other name: extracting biological names. J of Biomedical Informatics 2002, 35(4):247-259.

21. Tuason O, Chen L, Liu H, Blake JA, Friedman C: Biological Nomenclatures: A Source of Lexical Knowledge and Ambiguity. Proc Pacific Symp on Biocomputing 2004, 238-249.

22. Hsu CN, Chang YM, Kuo CJ, Lin YS, Huang HS, Chung IF: Integrating High Dimensional Bi-directional Parsing Models for Gene Mention Tagging Bioinformatics 2008, 24(ISMB2008):i286-i294.

23. Wei $\mathrm{CH}$, Huang $\mathrm{IC}$, Hsu YY, Kao HY: Normalizing Biomedical Name Entities by Similarity-Based Inference Network and De-ambiguity Mining. Ninth IEEE International Conference on Bioinformatics and Bioengineering Workshop: Semantic Biomedical Computing: 2009; Taichung, Taiwan 2009, 461-466.

24. Lu Z, Wilbur WJ: Overview of BioCreative III Gene Normalization. BioCreative III Workshop Maryland,Bethesda; 2010.

25. Carroll HD, Kann MG, Sheetlin SL, Spouge JL: Threshold Average Precision (TAP-k): A Measure of Retrieval Designed for Bioinformatics. Bioinformatics 2010, 26(14):1708-1713.

26. Settles B: ABNER: an open source tool for automatically tagging genes, proteins and other entity names in text. Bioinformatics 2005, 21(14):3191-3192.

27. Kim JD, Ohta T, Tateisi Y, Tsujii J: GENIA corpus-a semantically annotated corpus for bio-textmining. Bioinformatics 2003, 19(Suppl. 1):i180-i182.

doi:10.1186/1471-2105-12-S8-S5

Cite this article as: Wei and Kao: Cross-species gene normalization by species inference. BMC Bioinformatics 2011 12(Suppl 8):S5.

\section{Submit your next manuscript to BioMed Central and take full advantage of:}

- Convenient online submission

- Thorough peer review

- No space constraints or color figure charges

- Immediate publication on acceptance

- Inclusion in PubMed, CAS, Scopus and Google Scholar

- Research which is freely available for redistribution 